\title{
The importance of buprenorphine research in the opioid crisis
}

\author{
Sarah A. Pendergrass $\mathbb{D}^{1} \cdot$ Richard C. Crist ${ }^{2} \cdot$ Laney K. Jones ${ }^{3} \cdot$ Jason R. Hoch $^{4} \cdot$ Wade H. Berrettini $^{2,5}$
}

Received: 17 September 2018 / Revised: 15 October 2018 / Accepted: 14 November 2018 / Published online: 7 January 2019

(c) Springer Nature Limited 2019

\begin{abstract}
With the urgency to treat patients more effectively for opioid use disorder in the midst of the opioid epidemic, a key area for precision medicine is to improve individualized medication-assisted treatment for opioid use disorder. The expansion of medication-assisted treatment is a key to reducing illicit opioid use, preventing opioid overdose deaths, and reducing the comorbidities and societal impacts of opioid use disorder. The most common medication for opioid use disorder will soon be buprenorphine. Research to date shows the successful impact of buprenorphine treatment, including the pharmacogenomics of buprenorphine response and treatment efficacy. Buprenorphine is also a promising treatment for depression and anxiety, and neonatal opioid withdrawal syndrome (NOWS). However, the rates of success with medication-assisted treatment for opioid use disorder, particularly at the beginning of treatment, still show many individuals relapsing to illicit opioid use. With the scope of the opioid crisis, there is an urgent need for expansion of buprenorphine treatment research to provide critical information for improving outcomes of opioid use disorder. Implementing the best strategies for opioid use disorder treatment is of dire urgency and will save lives.
\end{abstract}

\section{Opioid use disorder and opioid substitution therapy}

The opioid addiction crisis is ongoing, with the majority of those with opioid use disorder (OUD) having started with prescription opioids [1]. Every day more than 115 people die from opioid-involved overdose in the US, with the greatest percentage of those deaths involving prescription opioids [2]. Beyond overdose, there are significant impacts from OUD, including the burden of addiction on individuals and society. These burdens include significant individual comorbidities, economic loss (e.g., emergency health care utilization [3]), impacts on children through neonatal opioid

Sarah A. Pendergrass

spendergrass@geisinger.edu

1 Biomedical and Translational Informatics Department, Geisinger, Danville, PA, USA

2 Center for Neurobiology and Behavior, Perelman School of Medicine, University of Pennsylvania, Philadelphia, PA, USA

3 Center for Pharmacy Innovation and Outcomes, Precision Health Center, Geisinger, Forty Fort, PA, USA

4 Department of Psychiatry and Addiction Medicine, Neurosciences Institute, Geisinger, Danville, PA, USA

5 Geisinger, Danville, PA, USA withdrawal syndrome (NOWS) [4], the destructive effect of OUD on families, and impact within the U.S. legal and criminal justice system [5]. Many medical providers are now prescribing opioid-free treatment modalities (particularly for chronic pain) to prevent future addiction to opioids; however, there remain millions of people affected by OUD. OUD is a chronic disease that can be compared to other chronic health conditions, such as diabetes and heart failure. Thus treatment for OUD is now being considered for extended periods [6].

Research has shown that treating OUD through medicationassisted treatment (MAT) with opioid substitution therapy (OST) leads to decreased mortality rates and improved outcomes [7]. The current FDA-approved treatments for OUD frequently consist of OST with medications, such as methadone and buprenorphine. OST medications act as agonists at the mu-opioid receptor (MOR), which is the primary target of addictive opioids. Methadone is a full MOR agonist. Buprenorphine is both a partial MOR agonist and a kappa-opioid receptor (KOR) antagonist.

\section{Opioid substitution therapy through buprenorphine}

Buprenorphine is quickly becoming the most important OST for OUD, because of its availability in primary care 
settings, accessibility for patients, and more favorable safety profile [8]. In the United States, buprenorphine is frequently compounded in sublingual tablets with naloxone to discourage patients from misusing the crushed tablet. The medication does not require daily observed dosing like methadone and is available in extended release formulations. All these factors can reduce access hurdles for some patients to OST and support greater adherence to therapy. However, a considerable limitation in access to buprenorphine-based OST remains due to the number of physicians who can prescribe buprenorphine. A Drug Enforcement Agency (DEA) waiver is available for the prescribing of buprenorphine to treat OUD. Clinicians have to complete 8 hours of training to obtain the waiver to prescribe buprenorphine for OUD. Mid-level practitioners, such as physician assistants and nurse practitioners, must have 24 hours of training. Thus, there are limitations for clinicians with already full schedules due to the time requirements for obtaining the waiver. There are other barriers, including concerns from providers about not having the skills to treat OUD even if cleared to prescribe buprenorphine, concerns about the lack of psychosocial and mental health support available for OUD patients, misconceptions about the value of OST, lack of training in addiction treatment, and misconceptions about addiction [9, 10]. It is also important to note another caveat to the improved accessibility of buprenorphine by patients, as there are health disparities in access to buprenorphine. For example, individuals in predominantly white, higherincome areas of New York City as of 2007 were more likely to have access to buprenorphine than low-income, predominantly African-American and Hispanic areas [11].

\section{Improving successful opioid substitution therapy}

While the treatment of OUD with OST improves outcomes compared to no treatment, there is a wide range of the overall success of OST, depending on what time period is being reported. Two recent reports indicated that $\sim 40 \%$ of patients treated with buprenorphine were retained in OST treatment for 1 year $[12,13]$. There are some known demographic, socioeconomic, and psychosocial contributors to the retention of individuals in MAT, based mostly on research in methadone-based OST. These contributors include depression, stress, employment status, association with other drug users, and stability of personal relationships [14]. Schizophrenia, bipolar disorder, and alcohol abuse or dependence have also been found to predict relapse rates during treatment [15]. Anxiety has been associated with opioid positive urine drug screens during methadone OST [16]. Anxiety and substance use, specifically alcohol and benzodiazepines, have also been identified as predictors of relapse during buprenorphine treatment [17]. In one study of buprenorphine treatment, dropout was associated with age, ethnicity, hepatitis C infection, and employment status [18].

Precision medicine characterizes individual variability in genes and environment for improving individualized treatment response. Precision medicine principles can be applied in order to improve OST/MAT outcomes. Expanding our understanding of the key factors that contribute to OST success for each patient, including psychological, socioeconomic, environmental, and genetic factors, in longitudinal research provides a way to better target for each patient the level and types of support most critical to successful OST adherence. No study thus far has investigated the impact of combinations of these factors on the efficacy or effectiveness of OST in a real-world setting. Precision medicine will help identify if other medications (e.g. methadone or naltrexone), increased patient monitoring, and/or employing different behavioral therapies will better support successful OST and MAT for specific patients at increased risk of relapse. Further, through the use of longitudinal clinical data, there is an opportunity for better predictions of the long-term health outcomes of individuals.

Precision medicine also includes the use of genetic data, through genetic biomarkers that correlate with increased or decreased risk of outcomes. It will be important to use pharmacogenomic analyses in buprenorphine treatment to identify genetic variation that corresponds with efficacy in treatments for OUD. Efficacy can be defined as either reduction in drug use or prevention of relapse, and there are a series of studies that have used this pharmacogenomics approach in the treatment of nicotine and alcohol use disorders [19-25]. Several studies have also identified variants associated with either the dose or serum concentration of methadone [26-32]. Metabolism of buprenorphine is variable among individuals suggesting a similar genetic etiology. Buprenorphine is metabolized by CYP450 enzymes, and research has well characterized the impact of genetic variation for CYP450 enzymes for other drug response [33-35]. Genetic variation impacting pharmacokinetics and pharmacodynamics for buprenorphine will likely be important for treatment outcomes, although there is no published research identifying polymorphisms associated with buprenorphine dose or serum concentration [36]. There have been some studies of the pharmacogenetics of buprenorphine efficacy, implicating OPRD1, SLC6A3, SLC6A4, and COMT; however, far more research must be done in larger sample sizes for further discovery [23, 24, 37, 38]. There is also the unexplored area of the pharmacogenomics of adverse reactions to buprenorphine [36]. Thus, there is a great potential for determining prospective genetic biomarkers to identify patients who will benefit the most from 
buprenorphine-based OST, and, conversely, patients at greatest risk of relapse who would benefit from additional treatment strategies to support recovery. It is important to note that the pharmacogenomics research to date has been in those of both European and African ancestry, and that ongoing research needs to continue across multiple ancestries, including expanding across Hispanics and other ethnic groups, so that pharmacogenomic biomarkers are well characterized across ancestry.

\section{Resources for improving precision medicine for buprenorphine-based ost}

To obtain sufficient cohorts of patients for expanded research into buprenorphine-based OST, a valuable resource will be health care systems with longitudinal electronic health record data (EHR) data and, when possible, these EHRs coupled with genetic data. There is existing research showing the utility of using clinical data for addiction research. For instance, patients' clinical data have shown relevant changes up to 2 years before opioid overdose, which supports using the longitudinal clinical data of patients for relapse prediction [39]. Vivolo-Kantor et al. characterized the rates of overdose using emergency department data and hospital billing data from July 2016 to September 2017, noting increase in overdose across that time period as well as the variability in location of these events, varying by region and urbanization level [40]. Hasegawa et al. evaluated emergency department visits for opioid overdose [41]. They determined that frequent emergency department visits were associated with higher likelihood of future hospitalizations and near-fatal events. These are examples of information that could be used within health care systems to identify individuals with greatest need for intervention, potentially intervening far before overdose events. Further, research has shown that buprenorphine treatment started in the emergency room, with a direct 'warm handoff' to MAT clinics, increases engagement in formal addiction treatment [42]. For many patients this was their first treatment contact.

There are specific health system characteristics, that if present, will support the discovery of the most robust insights into key contributors to successful OST and support productive largescale research. This includes health systems that have a substantial commitment to high-quality MAT for OUD, with psychosocial supports of drug counseling and case management encouraging continued patient retention. Health care systems with a decade or more of EHR data across primary and speciality care are also important, as EHR data provides inroads to multifaceted analyses of patient characteristics without time- and resource-consuming data collection. Health care systems that have digitally accessible ways of obtaining more information from patients, such as web portals for collecting patient responses, are also critical for facilitating collection of additional highly structured questionnaire data to further improve patient characterization for research. Medical systems taking care of large stable populations of OUD patients of diverse ancestries, as well as a wide range of ages including pregnant women and children, are possible within areas hardest hit in the US with the opioid epidemic. Health care systems with strong research infrastructure, including biorepositories, also provide a further strength for robust OST research. Other features that can contribute to robust research include embedded psychiatry residents cross-training within MAT clinics, with full time pharmacist involvement and system oversight. Health care systems with close relationships to local organizations are also important, as they are well connected to the larger environment in which patients are treated. This includes drug and alcohol counseling agencies, community mental health providers, county authorities, law enforcement, and pharmacies, which are integral to successful OST. Warm hand-offs of patients in general, including incorporating obstetric providers for concurrent MAT and pre-natal care, will also capture more data from OUD patients before and during OST.

\section{An example health-care system for expanding buprenorphine OST research}

One health care system that meets most of these criteria for expanded precision medicine research in OST is Geisinger, the largest health care provider in central Pennsylvania, which has now expanded into New Jersey. Geisinger is a primary and specialty care provider, and implemented an EHR in 1996. The Geisinger patient population is geographically stable, so longitudinal EHR data are common in this health system. Pennsylvania is one of the states most impacted by the opioid abuse epidemic across the United States: at least 10 Pennsylvanian's die every day from opioid overdose [43]. Hospital admissions for opioid overdose have nearly quadrupled since 2010 in Pennsylvania.

There have been limited numbers of addiction support providers in rural areas, such as the rural central Pennsylvania areas covered by Geisinger. There are also few primary care practitioners with training in addiction treatment. To address the opioid epidemic, Geisinger has opened three MAT clinics in 2017-18, with a fourth to soon be opened. In the first 8 months of 2018, 848 unique OUD patients were treated in these MAT clinics. Geisinger uses a hub and spoke model for deploying MAT clinics [44], where a centralized addiction specialist team works with primary care practitioners acting as "spokes". With this structure, there can be a rapid expansion of access to high-quality MAT, including quantitative buprenorphine and 
buprenorphine metabolite monitoring, referral and followup with psychosocial supports from licensed drug and alcohol counselors, primary care physician support, structured prescribing intervals, rigorous diversion control policies, and pharmacist oversight for contraindicated medications. The MAT clinics employ witnessed dosing of buprenorphine with serial drug level monitoring for diversion control. Geisinger also is pursuing the eventual elimination of co-prescribing of contraindicated and concerning abusable medication, with a $90 \%$ reduction of this kind of prescribing. The hub and spoke model addresses many of the hurdles described for primary care providers by providing resources for patients and their primary providers beyond OST prescriptions. These MAT clinics can reduce the number of overdose deaths for treated patients, minimize the risk of diversion, and decrease the risk of relapse.

Geisinger MAT clinics provide comprehensive addiction evaluations, including use of the Addiction Severity Index embedded in the EHR, a critical asset for precision medicine OST research. Additionally, withdrawal assessment for alcohol (CIWA), and clinical opiate withdrawal scale (COWS) scores are recorded. This information is being integrated into the EHR at the beginning of care, and then updated every 6 months. These EHR data support logical prescribing levels for buprenorphine, critical measures of patient acuity, and provide information flow between the hub of the MAT treatment and the spokes (primary care providers and addiction treatment coordinators) via the EHR. These EHR data are thus a valuable opportunity for de-identified research within these records.

In terms of genetic data, the MyCode Community Health Initiative at Geisinger is a biorepository that will have more than 200,000 individuals with whole-exome sequencing and whole-genome array based genotyping in parallel. There is already a focus on consenting patients for MyCode within the MAT clinics for expanding the use of genomic data in addiction research. This genetic data coupled with the data collected as a part of general patient care as well as within addiction treatment at Geisinger will be a rich resource for pharmacogenomic analyses of buprenorphine response and adverse events.

Other health system resources with these optimal characteristics may also include the Kaiser Permanente, the Million Veteran Program and Vanderbilt University Medical Center. This list is not meant to be exhaustive, but illustrative.

\section{Improving translation of research findings to clinical practice}

While it is critical to expand research into key factors to improve precision medicine of OST, it is also important to identify the best strategies for bringing research findings to addiction care so that information that can improve patient outcomes can be utilized more quickly. The translation from discovery in research to updates in clinical practice is often slow, taking upwards of 17 years [45]. The implementation of pharmacogenomic findings in general into clinical care of patients has been slow [46]. With the ongoing OUD crisis, it is critical to develop an understanding now of the best strategies for widespread dissemination of interventions [47]. Methods of implementation science can be used to understand barriers and facilitators to implementation into care, and better understand organizational readiness to implement change. By applying implementation science, discovery of useful pharmacogenomics and clinical information can be quickly incorporated into clinical care, ensuring the success of bringing precision medicine principles to OST for successful MAT. Patients' and healthcare professionals' positive attitudes and beliefs toward utilizing and receiving pharmacogenomic results have been well-documented [48-50]. What are not known are the factors that facilitate and hinder a healthcare system's ability to successfully implement pharmacogenomic testing and the use of other clinical and demographic factors to improve the care of patients undergoing OST with buprenorphine. This is a beneficial area of research.

\section{Expansion of buprenorphine for treatment of mood and anxiety disorders}

In addition to the importance of elucidating the role of buprenorphine as OST, and the importance of precision medicine for buprenorphine treatment, there is also an important area of research to be pursued in better understanding the antidepressant and anxiolytic effects of buprenorphine, and the impact of those factors on therapeutic response. There are high rates of co-morbidity of OUD with anxiety and mood disorders, and the antidepressant effects of buprenorphine at low doses may prove critical in improving OST and MAT outcomes, since depression is associated with continued use during treatment [14].

There are now are multiple preclinical rodent studies which demonstrate that chronic administration of relatively low doses of buprenorphine have antidepressant and anxiolytic effects [51-54]. This effect of buprenorphine is attributed to its high affinity kappa-opioid receptor (KOR) antagonism, as other KOR antagonists have similar effects [55]. The buprenorphine antidepressant activity in the forced swim test in mice is absent in OPRKI (the gene encoding KOR) gene null mutants [53]. However, in the novelty-induced hypophagia test in mice, the antidepressant effects of buprenorphine were absent in OPRM1 null mutants but 
retained in OPRK1 null mutants [56]. Thus, the buprenorphine MOR and KOR activities may both be relevant to its effects on rodent models of anxiety and depression.

In humans, a study by Bershad et al. found buprenorphine dampens the response to stress [57]. Trials of buprenorphine in depression are promising. In an open label, 8-week, flexible dose study of 15 older persons with treatment-resistant depression, Karp et al. [58] noted improvements over baseline depression ratings after 3 weeks of low dose buprenorphine, where daily doses ranged from 0.2 to $1.6 \mathrm{mg}$. In a double-blind, placebocontrolled trial, Fava et al. [59] reported significant improvements over baseline depression ratings among adults with treatment-resistant depression when treated with $2 \mathrm{mg}$ buprenorphine combined with a mu antagonist, samidorphan $(2 \mathrm{mg})$, as an add-on to existing antidepressant therapy. In subsequent phase III double blind trials, the Alkermes compound ALK 5461 (buprenorphine + samidorphan) showed promising antidepressant activity, resulting in an FDA new drug application by Alkermes. However, it is important to note that details of the trials have not yet been published in peer-reviewed format. It is noteworthy that the efficacious anxiolytic and antidepressant doses of buprenorphine are $\sim 1 / 10$ th the doses used to treat OUD.

With the promising evidence of the anxiolytic and antidepressant activity for buprenorphine, important research questions have been raised. For example, what are the buprenorphine transcriptomic and proteomic profiles that convey the antidepressant and anxiolytic activity? And are these profiles similar to those of serotonergic or noradrenergic antidepressants? Which neural pathways are involved in these activities of buprenorphine, and how similar are these pathways to those of serotonergic or noradrenergic antidepressants? Are there pharmacogenetic, biomarker, and or/clinical profiles of responders to the antidepressant and anxiolytic activity of buprenorphine?

\section{Buprenorphine treatment for neonatal opioid withdrawal syndrome}

Another important area of research is the treatment of neonatal opioid withdrawal syndrome (NOWS), a series of conditions newborns have when going through withdrawal from opioids they were exposed to in the womb. Kraft et al. [60] documented that buprenorphine was a superior treatment for NOWS, compared to the standard treatment of morphine. In their double blind clinical trial, 63 term infants with NOWS were randomized. The buprenorphine-treated group $(n=33)$ had a shorter mean hospital stay (21 versus 33 days, $\quad p<0.001)$ and a shorter duration of pharmacotherapy for NOWS (15 versus 28 days, $p<0.001)$. It can be expected that buprenorphine will be adopted as a standard treatment for NOWS, given these highly significant benefits. There are a series of questions to be answered through research, which will be critical to using buprenorphine in treatment of NOWS. For example, what are the developmental effects of perinatal buprenorphine exposure, and how do they differ from those of morphine, short and long-term? What differences in outcomes do we identify in the use of buprenorphine for NOWS when compared to adult buprenorphine exposure? Are there key contributors to NOWS buprenorphine response, whether they be pharmacogenetic or clinical?

\section{Conclusions}

Buprenorphine is a critically useful medication in opioid crisis. Expanded precision medicine research into the environmental and genetic characteristics of patients most likely to benefit from buprenorphine treatment of OUD is urgently needed to improve therapeutic responses. Implementation research guiding health systems on mechanisms to bring these findings quickly into the clinic are equally important. If research expands and focuses in these areas there is the potential for improving long-term outcomes and saving lives. An additional promising area of investigation is precision medicine of the anxiolytic and antidepressant activities of buprenorphine. Understanding the antidepressant and anxiolytic aspects of buprenorphine may help reduce the suicide rate, by providing an alternative pharmacotherapy for treatment-resistant depression.

\section{Compliance with ethical standards}

Conflict of interest The authors declare no conflict of interest.

Publisher's note: Springer Nature remains neutral with regard to jurisdictional claims in published maps and institutional affiliations.

\section{References}

1. Kolodny A, Courtwright DT, Hwang CS, Kreiner P, Eadie JL, Clark TW, et al. The prescription opioid and heroin crisis: a public health approach to an epidemic of addiction. Annu Rev Public Health. 2015;36:559-74.

2. CDC Injury Center. Understanding the epidemic: Drug overdose. 2018. https://www.cdc.gov/drugoverdose/epidemic/index.html. Accessed 11 Sept 2018.

3. Florence CS, Zhou C, Luo F, Xu L. The economic burden of prescription opioid overdose, abuse, and dependence in the United States, 2013. Med Care. 2016;54:901-6.

4. Klaman SL, Isaacs K, Leopold A, Perpich J, Hayashi S, Vender J, et al. Treating women who are pregnant and parenting for opioid 
use disorder and the concurrent care of their infants and children: literature review to support national guidance. J Addict Med. 2017;11:178-90.

5. National Institute on Drug Abuse. Treating opioid addiction in criminal justice settings. https://www.drugabuse.gov/publications/ treating-opioid-addiction-in-criminal-justice-settings/treatingopioid-addiction-in-criminal-justice-settings. Accessed 11 Sept 2018.

6. Fiellin DA, Schottenfeld RS, Cutter CJ, Moore BA, Barry DT, O'Connor PG. Primary care-based buprenorphine taper vs maintenance therapy for prescription opioid dependence: a randomized clinical trial. JAMA Intern Med. 2014;174:1947-54.

7. Sordo L, Barrio G, Bravo MJ, Indave BI, Degenhardt L, Wiessing $\mathrm{L}$, et al. Mortality risk during and after opioid substitution treatment: systematic review and meta-analysis of cohort studies. BMJ. 2017;357:j1550.

8. Wakeman SE, Barnett ML. Primary care and the opioid-overdose crisis - buprenorphine myths and realities. N Engl J Med. 2018. https://doi.org/10.1056/NEJMp1802741.

9. Andrilla CHA, Coulthard C, Larson EH. Barriers rural physicians face prescribing buprenorphine for opioid use disorder. Ann Fam Med. 2017;15:359-62.

10. Haffajee RL, Bohnert ASB, Lagisetty PA. Policy pathways to address provider workforce barriers to buprenorphine treatment. Am J Prev Med. 2018;54:S230-S242.

11. Hansen H, Siegel C, Wanderling J, DiRocco D. Buprenorphine and methadone treatment for opioid dependence by income, ethnicity and race of neighborhoods in New York City. Drug Alcohol Depend. 2016;164:14-21.

12. Shcherbakova N, Tereso G, Spain J, Roose RJ. Treatment persistence among insured patients newly starting buprenorphine/ naloxone for opioid use disorder. Ann Pharmacother. 2018;52:405-14.

13. Manhapra A, Agbese E, Leslie DL, Rosenheck RA. Three-year retention in buprenorphine treatment for opioid use disorder among privately insured adults. Psychiatr Serv Wash DC. 2018;69:768-76.

14. Brewer DD, Catalano RF, Haggerty K, Gainey RR, Fleming CB. A meta-analysis of predictors of continued drug use during and after treatment for opiate addiction. Addict Abingdon Engl. 1998;93:73-92.

15. Clark RE, Baxter JD, Aweh G, O'Connell E, Fisher WH, Barton BA. Risk factors for relapse and higher costs among medicaid members with opioid dependence or abuse: opioid agonists, comorbidities, and treatment history. J Subst Abus Treat. 2015;57:75-80.

16. Yin W, Pang L, Cao X, McGoogan JM, Liu M, Zhang C, et al. Factors associated with depression and anxiety among patients attending community-based methadone maintenance treatment in China. Addict Abingdon Engl. 2015;110:51-60.

17. Ferri M, Finlayson AJR, Wang L, Martin PR. Predictive factors for relapse in patients on buprenorphine maintenance. Am J Addict. 2014;23:62-67.

18. Weinstein ZM, Kim HW, Cheng DM, Quinn E, Hui D, Labelle CT, et al. Long-term retention in office based opioid treatment with buprenorphine. J Subst Abus Treat. 2017;74:65-70.

19. Anton RF, Oroszi G, O’Malley S, Couper D, Swift R, Pettinati H, et al. An evaluation of mu-opioid receptor (OPRM1) as a predictor of naltrexone response in the treatment of alcohol dependence: results from the combined pharmacotherapies and behavioral interventions for alcohol dependence (COMBINE) study. Arch Gen Psychiatry. 2008;65:135-44.

20. Chamorro A-J, Marcos M, Mirón-Canelo J-A, Pastor I, GonzálezSarmiento R, Laso F-J. Association of $\mu$-opioid receptor (OPRM1) gene polymorphism with response to naltrexone in alcohol dependence: a systematic review and meta-analysis. Addict Biol. 2012;17:505-12.
21. Lerman C, Wileyto EP, Patterson F, Rukstalis M, AudrainMcGovern J, Restine S, et al. The functional mu opioid receptor (OPRM1) Asn40Asp variant predicts short-term response to nicotine replacement therapy in a clinical trial. Pharm J. 2004;4:184-92.

22. Oslin DW, Berrettini W, Kranzler HR, Pettinati H, Gelernter J, Volpicelli JR, et al. A functional polymorphism of the mu-opioid receptor gene is associated with naltrexone response in alcoholdependent patients. Neuropsychopharmacol Publ Am Coll Neuropsychopharmacol. 2003;28:1546-52.

23. Clarke T-K, Crist RC, Ang A, Ambrose-Lanci LM, Lohoff FW, Saxon AJ, et al. Genetic variation in OPRD1 and the response to treatment for opioid dependence with buprenorphine in EuropeanAmerican females. Pharm J. 2014;14:303-8.

24. Crist RC, Clarke T-K, Ang A, Ambrose-Lanci LM, Lohoff FW, Saxon AJ. et al. An intronic variant in OPRD1 predicts treatment outcome for opioid dependence in African-Americans. Neuropsychopharmacology. 2013;38:2003-10.

25. Kranzler HR, Covault J, Feinn R, Armeli S, Tennen H, Arias AJ, et al. Topiramate treatment for heavy drinkers: moderation by a GRIK1 polymorphism. Am J Psychiatry. 2014;171:445-52.

26. Crettol S, Déglon J-J, Besson J, Croquette-Krokar M, Hämmig R, Gothuey I, et al. ABCB1 and cytochrome P450 genotypes and phenotypes: influence on methadone plasma levels and response to treatment. Clin Pharmacol Ther. 2006;80:668-81.

27. Hung C-C, Chiou M-H, Huang B-H, Hsieh Y-W, Hsieh T-J, Huang C-L, et al. Impact of genetic polymorphisms in ABCB1, CYP2B6, OPRM1, ANKK1 and DRD2 genes on methadone therapy in Han Chinese patients. Pharmacogenomics. 2011;12:1525-33.

28. Kharasch ED, Stubbert K. Role of cytochrome P4502B6 in methadone metabolism and clearance. J Clin Pharmacol. 2013;53:305-13.

29. Yang H-C, Chu S-K, Huang C-L, Kuo H-W, Wang S-C, Liu S-W, et al. Genome-wide pharmacogenomic study on methadone maintenance treatment identifies SNP rs17180299 and multiple haplotypes on CYP2B6, SPON1, and GSG1L associated with plasma concentrations of methadone $\mathrm{R}$ - and S-enantiomers in heroin-dependent patients. PLoS Genet. 2016;12:e1005910.

30. Levran O, Peles E, Hamon S, Randesi M, Adelson M, Kreek MJ. CYP2B6 SNPs are associated with methadone dose required for effective treatment of opioid addiction. Addict Biol. 2013;18:70916.

31. Levran O, O'Hara K, Peles E, Li D, Barral S, Ray B, et al. ABCB1 (MDR1) genetic variants are associated with methadone doses required for effective treatment of heroin dependence. Hum Mol Genet. 2008;17:2219-27.

32. Smith AH, Jensen KP, Li J, Nunez Y, Farrer LA, Hakonarson H, et al. Genome-wide association study of therapeutic opioid dosing identifies a novel locus upstream of OPRM1. Mol Psychiatry. 2017;22:346-52.

33. Crews KR, Gaedigk A, Dunnenberger HM, Leeder JS, Klein TE, Caudle KE, et al. Clinical pharmacogenetics implementation consortium guidelines for cytochrome P450 2D6 genotype and codeine therapy: 2014 update. Clin Pharmacol Ther. 2014;95:376-82.

34. PharmGKB. https://www.pharmgkb.org/. Accessed 1 Aug 2018.

35. Relling MV, Klein TE. CPIC: clinical pharmacogenetics implementation consortium of the pharmacogenomics research network. Clin Pharmacol Ther. 2011;89:464-7.

36. Crist RC, Clarke T-K, Berrettini WH. Pharmacogenetics of opioid use disorder treatment. CNS Drugs. 2018;32:305-20.

37. Gerra G, Somaini L, Leonardi C, Cortese E, Maremmani I, Manfredini M, et al. Association between gene variants and response to buprenorphine maintenance treatment. Psychiatry Res. 2014;215:202-7. 
38. Crist RC, Li J, Doyle GA, Gilbert A, Dechairo BM, Berrettini WH. Pharmacogenetic analysis of opioid dependence treatment dose and dropout rate. Am J Drug Alcohol Abus. 2018;44:431-40.

39. Maeng DD, Han JJ, Fitzpatrick MH, Boscarino JA. Patterns of health care utilization and cost before and after opioid overdose: findings from 10-year longitudinal health plan claims data. Subst Abus Rehabil. 2017;8:57-67.

40. Vivolo-Kantor AM, Seth P, Gladden RM, Mattson CL, Baldwin GT, Kite-Powell A, et al. Vital signs: trends in emergency department visits for suspected opioid overdoses-United States, July 2016-september 2017. MMWR Morb Mortal Wkly Rep. 2018;67:279-85.

41. Hasegawa K, Brown DFM, Tsugawa Y, Camargo CA. Epidemiology of emergency department visits for opioid overdose: a population-based study. Mayo Clin Proc. 2014;89:462-71.

42. D'Onofrio G, Chawarski MC, O'Connor PG, Pantalon MV, Busch SH, Owens PH. et al. Emergency department-initiated buprenorphine for opioiddependence with continuation inprimary care: outcomes during andafter intervention. J Gen Intern Med. 2017;32:660-6.

43. DEA and partners release 2016. Pennsylvania drug overdose death Report. https://www.dea.gov/press-releases/2017/07/27/dea-andpartners-release-2016-pennsylvania-drug-overdose-death-report. Accessed 11 Sept 2018.

44. Brooklyn JR, Sigmon SC. Vermont hub-and-spoke model of care for opioid use disorder: development, implementation, and impact. J Addict Med. 2017;11:286-92.

45. Green LW. Closing the chasm between research and practice: evidence of and for change. Health Promot J Austr. 2014;25:25-29.

46. Mills R, Voora D, Peyser B, Haga SB. Delivering pharmacogenetic testing in a primary care setting. Pharm Pers Med. 2013;6:105-12.

47. Brownson RC, Jacobs JA, Tabak RG, Hoehner CM, Stamatakis KA. Designing for dissemination among public health researchers: findings from a national survey in the United States. Am J Public Health. 2013;103:1693-9.

48. Patel HN, Ursan ID, Zueger PM, Cavallari LH, Pickard AS. Stakeholder views on pharmacogenomic testing. Pharmacotherapy. 2014;34:151-65.

49. Rogausch A, Prause D, Schallenberg A, Brockmöller J, Himmel W. Patients' and physicians' perspectives on pharmacogenetic testing. Pharmacogenomics. 2006;7:49-59.
50. Haga SB, O’Daniel JM, Tindall GM, Lipkus IR, Agans R. Survey of US public attitudes toward pharmacogenetic testing. Pharm J. 2012;12:197-204.

51. Carr GV, Bangasser DA, Bethea T, Young M, Valentino RJ, Lucki I. Antidepressant-like effects of kappa-opioid receptor antagonists in Wistar Kyoto rats. Neuropsychopharmacology. 2010;35:752-63.

52. Browne CA, van Nest DS, Lucki I. Antidepressant-like effects of buprenorphine in rats are strain dependent. Behav Brain Res. 2015;278:385-92.

53. Falcon E, Maier K, Robinson SA, Hill-Smith TE, Lucki I. Effects of buprenorphine on behavioral tests for antidepressant and anxiolytic drugs in mice. Psychopharmacol (Berl). 2015;232:90715.

54. Almatroudi A, Husbands SM, Bailey CP, Bailey SJ. Combined administration of buprenorphine and naltrexone produces antidepressant-like effects in mice. J Psychopharmacol Oxf Engl. 2015;29:812-21.

55. McLaughlin JP, Marton-Popovici M, Chavkin C. Kappa opioid receptor antagonism and prodynorphin gene disruption block stress-induced behavioral responses. J Neurosci J Soc Neurosci. 2003;23:5674-83.

56. Robinson SA, Erickson RL, Browne CA, Lucki I. A role for the mu opioid receptor in the antidepressant effects of buprenorphine. Behav Brain Res. 2017;319:96-103.

57. Bershad AK, Jaffe JH, Childs E, de Wit H. Opioid partial agonist buprenorphine dampens responses to psychosocial stress in humans. Psychoneuroendocrinology. 2015;52:281-8.

58. Karp JF, Butters MA, Begley AE, Miller MD, Lenze EJ, Blumberger DM, et al. Safety, tolerability, and clinical effect of lowdose buprenorphine for treatment-resistant depression in midlife and older adults. J Clin Psychiatry. 2014;75:e785-793.

59. Fava M, Memisoglu A, Thase ME, Bodkin JA, Trivedi MH, de Somer M, et al. Opioid modulation with buprenorphine/samidorphan as adjunctive treatment for inadequate response to antidepressants: a randomized double-blind placebo-controlled trial. Am J Psychiatry. 2016;173:499-508.

60. Kraft WK, Adeniyi-Jones SC, Chervoneva I, Greenspan JS, Abatemarco D, Kaltenbach K, et al. Buprenorphine for the treatment of the neonatal abstinence syndrome. $N$ Engl J Med. 2017;376:2341-2348. 